\title{
Solving THE BINARIZATION CHALLENGES IN DOCUMENT IMAGES USING OTSU MultileVEL
}

\author{
Enas M. Elgbbas, Mahmoud I. Khalil, Hazem Abbas \\ Faculty of Engineering, Ain Shams University, Cairo, Egypt
}

\begin{abstract}
This paper introduces a method for binarization of historical document images that suffer from non-uniform background, faint text, low contrast, stain, bleed-through, or shadow challenges. The proposed method adaptively detects the non-uniform background in the document image and eliminates it. Areas that contain missing text are adaptively identified and reprocessed separately. Stain and bleed-through objects are found depending on stroke width and locally binarized. Shadow is detected based on the image contrast. Otsu multilevel is applied for binarization. DIBCO series is used for testing.
\end{abstract}

\section{KEYWORDS}

Document image binarization, Otsu multilevel

\section{INTRODUCTION}

Document image binarization is a substantial stage in the image recognition and analysis. It is used to segment the pixels into text and background. Text and background represented by 0 and 1 , respectively. Text extraction may be challenging because of the noise in document images (such as non-uniform background, shadows, bleed-through, and stain) and faint text. Focus on noise elimination may cause loss of faint text. On the contrary, faint or low contrast text extraction may produce noisy images. Therefore, it is important to remove degradation of the document image while preserving meaningful information. Effectiveness of binarization method is known by evaluation its output, such that binarization of good quality document image is easier than degraded document image. The objective of this paper is to propose a binarization method for all types of degradation. This is done by suggesting a method to detect each image problem and solve it separately.

\section{LiTERATURE SURVEY}

In literature, many techniques were introduced for document binarization. Bataineh et al. [1] method depends on the contrast. It fails in compound challenges. Bataineh et al. [2] method depends on mean, standard deviation and the maximum level of the image. It fails if the local window is applied on the text body. In Lu [3] method, an iterative polynomial smoothing procedure is used for background estimation. It fails in bleeding through problem. Moghaddam 
[4] combines grid-based modeling and the estimated background. Shi [5] uses the stroke width. Faint text cannot be recovered, and stain or shadow cannot be removed. Nafchi [6] method is based on phase congruency and feature maps. Su [7] depends on the contrast. Faint text cannot be recovered in bright images. Howe [8] uses Laplacian and Canny filters, and the graph cut. Noise and misses in the text are exist. Jia [9] method votes multiple thresholds for pixels classification. Vo [10] uses a hierarchical deep network architecture. He fails to recover thin or weak strokes.

AL-Khatatneh [11] method depends on the standard deviation. It is used for low uneven illumination document images. In Kefali [12] method, global thresholding classified the most pixels, and the remaining pixels are processed depending on their neighborhood information. This method misclassified the dark background or light foreground. In Jetley [13] method, gray scale image is processed using the Kittler [14] method at multiple scales. Then text line segmentation approach is used. It is robust to slightly degraded images. Pai [15] determines a threshold value using intelligent block size. It failed in very low or high illuminated document. We conclude that no binarization technique is completely accurate for all document image challenges. The objective of this paper is to propose a binarization method for solving the compound challenges. This paper is presented as follow; in Section 3, the proposed method is explained. Experimental results and conclusions are presented in Sections 4 and 5, respectively.

\section{ThE Proposed MeThod}

In the proposed method, the background of the investigated image is initially estimated and inspected. If there is a non-uniform background, it is detected and eliminated. Subsequently, a global binarization method is applied to produce a binary image used in the stork width estimation and contrast calculation. The area that contains the missing text is located in the gray scale image using the estimated background and locally recovered based on the stock width and the text contrast. Furthermore, stain and bleed-through objects are detected depending on the estimated stroke width. Then, they are locally binarized to extract text. The calculated contrast is used in shadow detection.

\subsection{NON-UNIFORM BACKGROUND ELIMINATION}

For background estimation, an initial foreground $(f)$ is estimated by applying Otsu [16] on an obtained high contrast image $(H C)$. $\mathrm{f}$ is applied as a mask on the gray scale image $(I)$. So that, the background intensity mean $\left(B G_{\text {mean }}\right)$ can be calculated. Text pixels in the gray scale image are replaced by the mean. Eq.1 gives the estimated backgrounds $(B G)$

$$
B G(x, y)=\left\{\begin{array}{c}
B G_{\text {mean }}, f(x, y)=0 \\
I(x, y), \quad \text { Otherwise }
\end{array}\right.
$$

Figure 1 shows background estimation. Figure 1a and Figure 1b show the grayscale images. Figure 1c and Figure 1d are the high contrast images. Figure 1e and Figure 1f are the initial foregrounds. Figure 1g and Figure 1h show the estimated background.

For non-uniform background detection, the estimated background is divided into three segments $(\mathrm{S}=1,2,3)$ using Otsu multilevel method [16]. Figure 2a and Figure 2b show the three segments 
that are used to extract the darkest $\left(B G_{d}\right)$, the fuzzy $\left(B G_{f}\right)$ and the brightest $\left(B G_{b}\right)$ background regions. $B G_{d}$ given by Eq.(2) may contain faint text that cannot be extracted in initial foreground as shown in Figure 2c, or it may be due to the presence of non-uniform background or shadow as shown in Figure 2 d. $B G_{f}$ given by Eq.(3) is shown in Figure 2e and Figure 2 f. $B G_{b}$ given by Eq.(4) is a pure background with completely extracted text in the initial foreground as shown in Figure $2 \mathrm{~g}$ and Figure $2 \mathrm{~h}$.

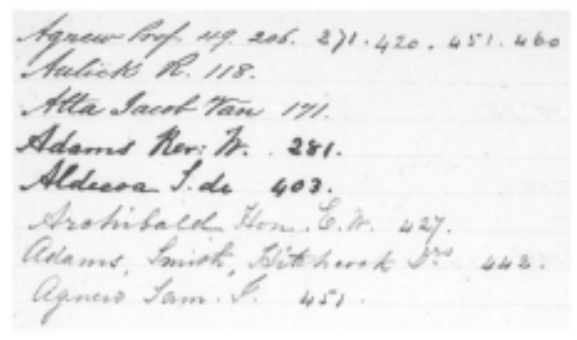

(a)

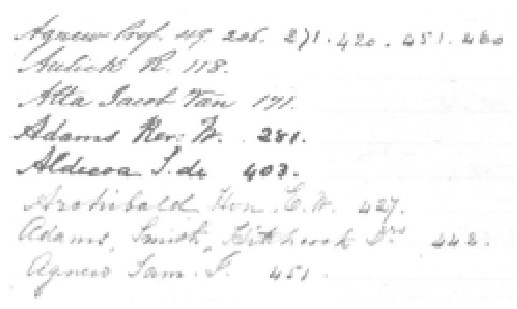

(c)

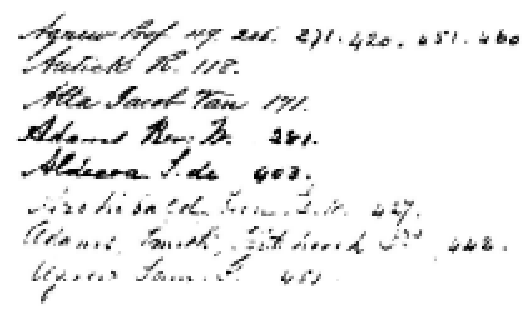

(e)

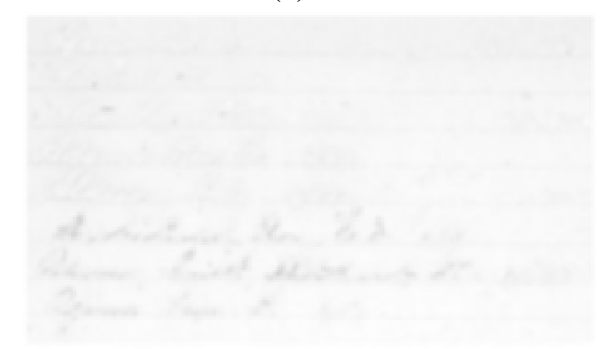

(g)

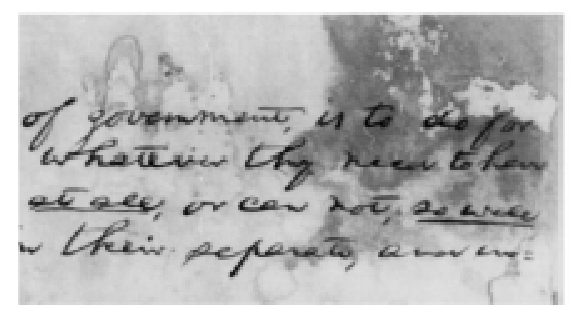

(b)

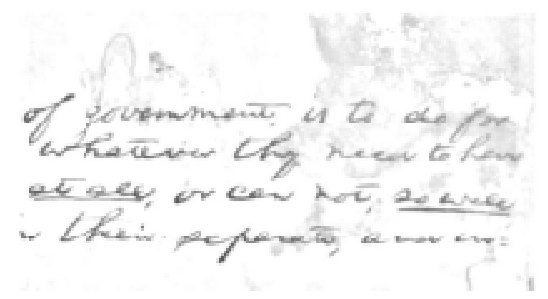

(d)

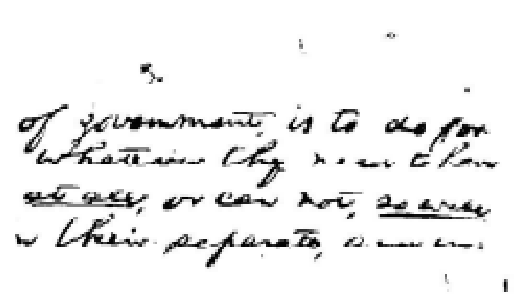

(f)

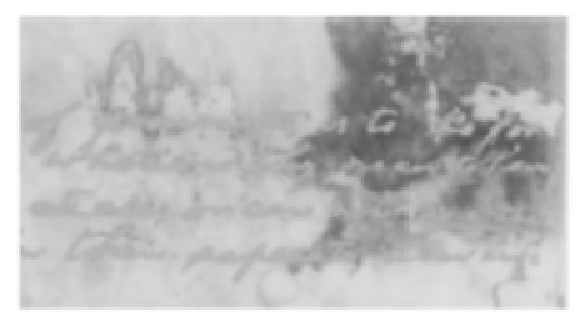

(h)

Figure 1. Background estimation: (a), (b) The gray scale images. (c), (d) The high contrast images. (e), (f) The initial foregrounds. (g), (h) The estimated background 


$$
\begin{aligned}
& B G_{d}(x, y)=\left\{\begin{array}{c}
B G(x, y), S(x, y)=1 \\
255, \quad \text { Otherwise }
\end{array}\right. \\
& B G_{f}(x, y)=\left\{\begin{array}{c}
B G(x, y), S(x, y)=2 \\
255, \quad \text { Otherwise }
\end{array}\right. \\
& B G_{b}(x, y)=\left\{\begin{array}{cc}
B G(x, y), & S(x, y)=3 \\
255, & \text { Otherwise }
\end{array}\right.
\end{aligned}
$$

The intensity means for $B G_{d}, B G_{f}$, and $B G_{b}$ are calculated, producing $M_{d}, M_{f}$ and $M_{b}$, respectively. The difference between $M d$ and $M b$ produces high values in the images with nonuniform background. So, it is used as an indicator to the presence of non-uniform background. Image in Figure 1a has low $M_{\text {diff }}$, so it has uniform background. Image in Figure $1 \mathrm{~b}$ has high $M_{\text {diff, }}$, so it has non uniform background.

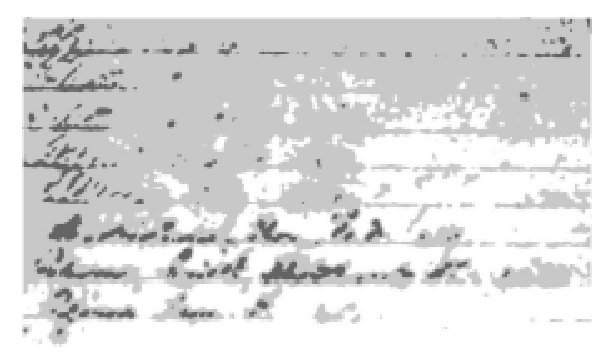

(a)

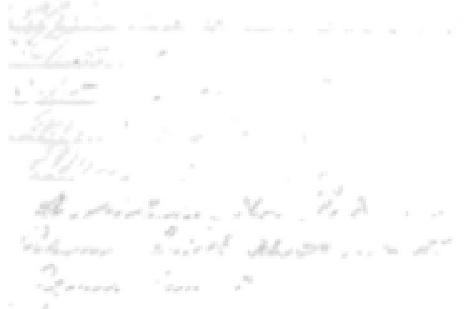

(c)

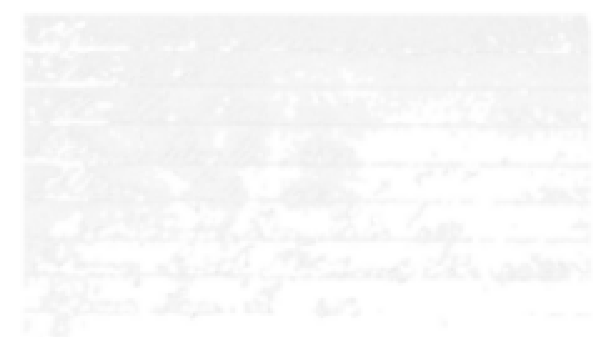

(e)

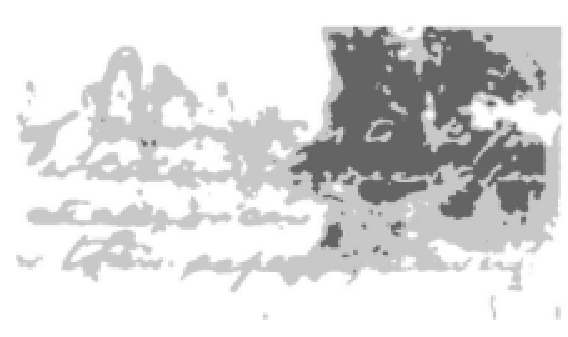

(b)

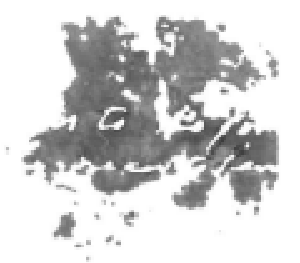

(d)

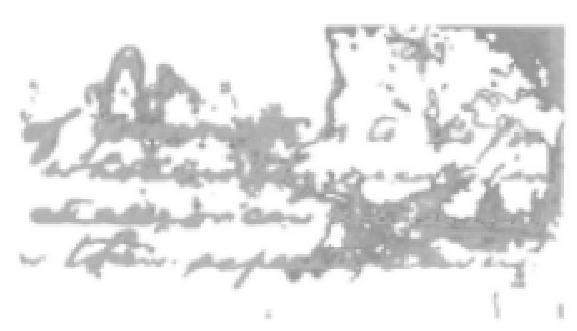

(f) 


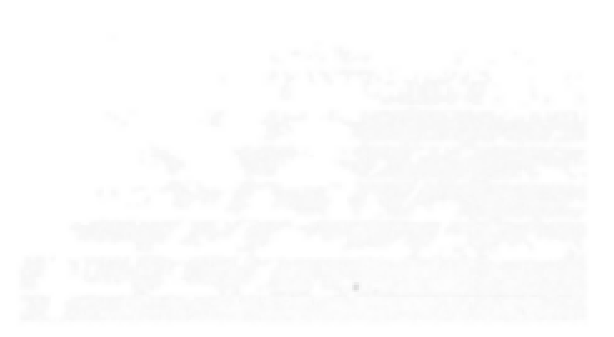

(g)

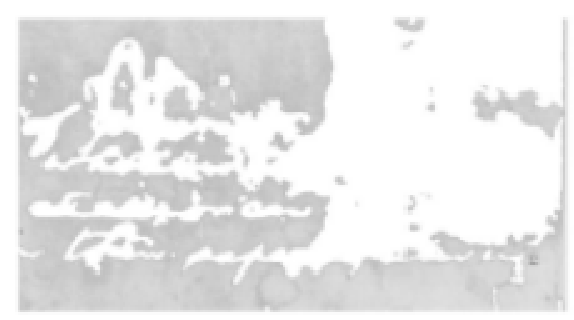

(h)

Figure 2. Dividing the estimated background: (a), (b) are the three segments. (c), (d) are the darkest background regions. (e), (f) are the fuzzy background regions. (g), (h) are the brightest background regions

The detected non-uniform background is removed by normalization as follow. The darkest region in the gray scale image (R1) given by Eq.(5), where $\mathrm{i}=1$, is adjusted to $\mathrm{R} 1_{\text {adj }} . M_{d}$ and 0 intensities are mapped to $M_{b}$ and the difference between $M_{b}$ and $M_{d}$ intensities, respectively. Adjustment of R1 is shown in Figure 3a and Figure 3b. Furthermore, the fuzzy region in the gray scale image (R2) given by Eq.(5), where $\mathrm{i}=2$, is adjusted $\mathrm{R} 2_{\text {adj }} . M_{f}$ and 0 are mapped to $M_{b}$ and the difference between $M_{b}$ and $M_{f}$, respectively. Adjustment of $R 2$ is shown in Figure $3 \mathrm{c}$ and Figure 3d. The normalized image given by Eq.6 is shown in Figure 3e.

$$
\begin{gathered}
R i_{[i=1,2]}(x, y)=\left\{\begin{array}{c}
I(x, y), S(x, y)=i \\
255, \quad \text { otherwise }
\end{array}\right. \\
\text { Nor }(x, y)=\left\{\begin{array}{c}
R \mathbf{1}_{\text {adj }}(x, y), S(x, y)=\mathbf{1} \\
R 2_{\text {adj }}(x, y), S(x, y)=2 \\
I(x, y), \text { Otherwise }
\end{array}\right.
\end{gathered}
$$

Although the normalization process eliminates the non-uniform background and applying global Otsu produces binary images $(B I)$ with high precision. But some text may be lost because the text contrast in $\mathrm{R} 1_{\text {adj }}$ may be reduced, as shown in Figure $3 f$. Furthermore, applying Otsu for images with uniform background produces acceptable results for $B I$. But the faint characters may be lost as shown in Figure $3 \mathrm{~h}$. The lost text is recovered by the aid of stroke width for the binary image resulted from applying Otsu $(B I)$. Using a single stroke width value is not effective in documents with different font sizes. So stroke width $(S W)$ and its upper limit $(Q)$ are estimated by the aid of skeleton and contour of $B I$.

\subsection{LOST TEXT RECOVERING}

For lost text recovering; the areas that are more likely contain missing text, are identified adaptively. A pseudo-local version of Otsu multilevel method is applied to that area.

For uniform background images, $R 1$ may contain faint text, faint text and shadow, shadow, or faint text in low contrast image as shown in Figure 4. Faint text is recovered depending on the 
image contrast. In high contrast images where shadow may exist, the broken characters are completed by $R l$ binarization. Objects with the same $S W$ of $B I$ and inside or connected to the rectangles that fit objects in $B I$, are selected. Figure 5 and Figure 6 are two high contrast images contain faint text and shadow, respectively. The faint text for both images is recovered in the final image $(F I)$ and the shadow is avoided. In case of very small $S W$ (as shown in Figure 7), binarization of. $R 1$ produces discontinuous. So the missed text is completed by taking all objects. In low contrast images shown in Figure 8, it is difficult to differentiate between faint text and background texture. So that, $B I$ objects whose sizes are smaller than the dot in characters like (i), (j), (;), and (.) and outside the rectangles are rejected.

For non-uniform background images, the text lost during normalization is recovered by applying Otsu multilevel on R1 and getting the lowest $10 \%$ intensity as text. Figure 9 shows lost text recovering.

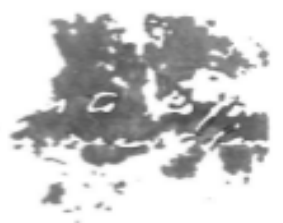

(a)

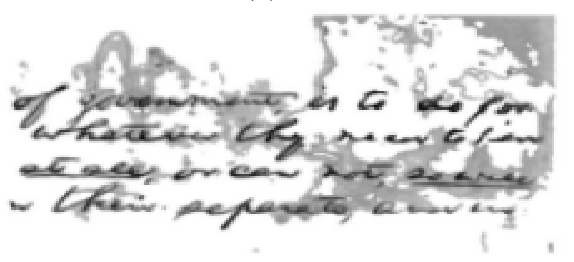

(c)

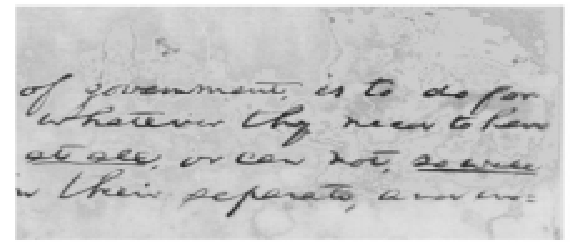

(e)

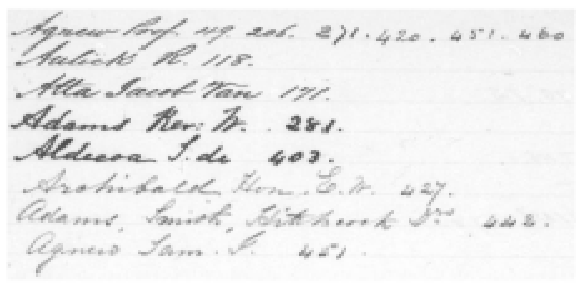

(g)

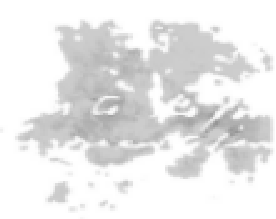

(b)

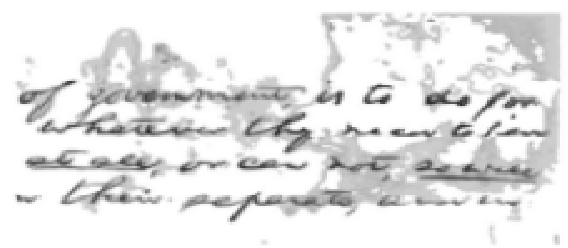

(d)

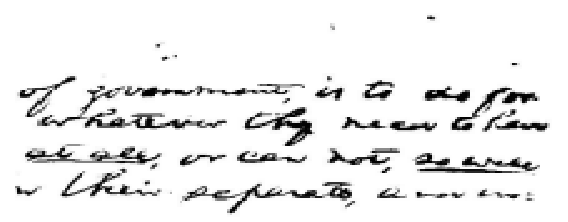

(f)

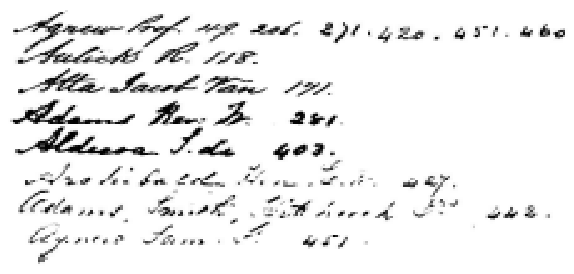

(h)

Figure 3. Obtaining the binary images after removing the non-uniform background: (a) R1. (b) R1 adj . (c) $\mathrm{R} 2$ (d)R $2_{\mathrm{adj}}$. (e) The normalized image. (f) The binary image for (e). (g) An uniform background image. (h) The binary image for $(\mathrm{g})$ 


\subsection{Stain And BleEding Through Detection}

Stain objects are located in BI by calculating the skeleton stroke width $(S S W)$, the vertical mean of the skeleton stroke width (VMSSW) and $Q$ for $B I$. By observing VMSSW, we found that stain objects exist where the $S S W$ exceeds five times of $Q$. These objects are selected in BI and applied as a mask to the gray scale image $(I)$. The obtained objects are binarized using Otsu multilevel method and getting the $33 \%$ lowest intensity as text. Figure 10 shows stain detection.

Bleed-through objects, that surrounded by noise and interference, are detected by comparing $Q$ for $\mathrm{f}$ (low noise) and $B I$. If it increased significantly, this means that there is background noise in the $B I$. So, objects with $S W$ greater than the $Q$ are selected in $B I$ and binarized using Otsu multilevel and getting the lowest $66 \%$ of intensity as text. Figure 11 shows bleeding through detection.

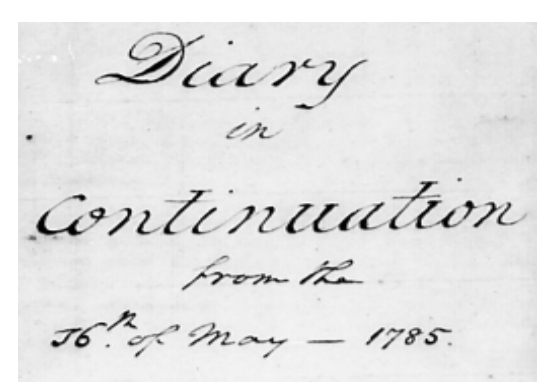

(a) $\mathrm{H} 04-12$

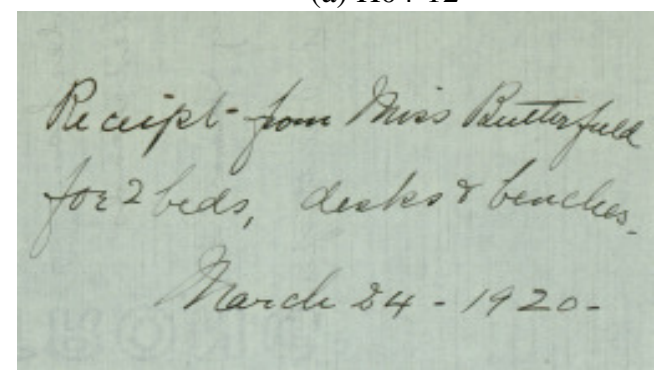

(c) HW2-11

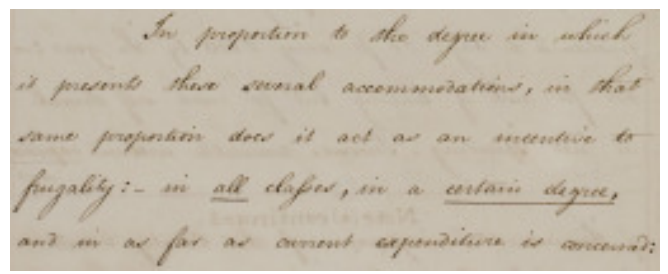

(e) $\mathrm{H} 01-14$



(g) $\mathrm{H} 13-12$



(b) R1

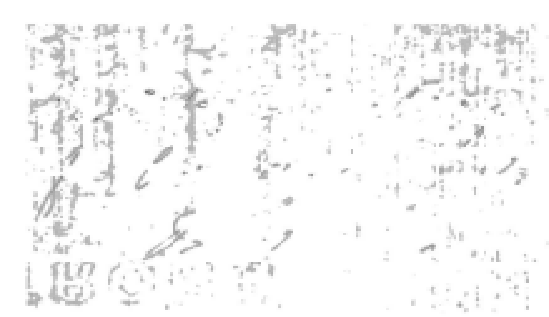

(d) Rl

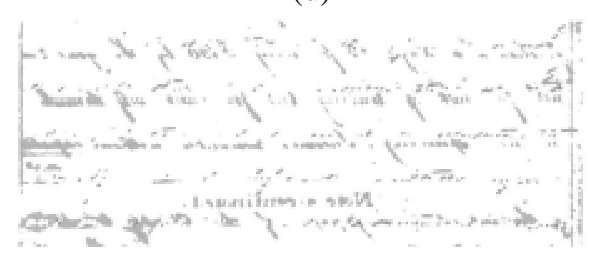

(f) $\mathrm{Rl}$

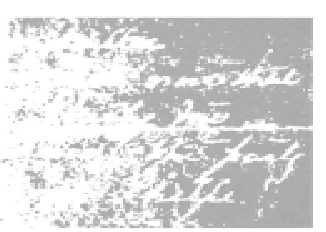

(h) Rl 
Figure 4. Challenges exist in R1 for the uniform background images: (a), (b) faint text. (c), (d) faint text and shadow.(e), (f) shadow. (g), (h) faint text in low contrast image.

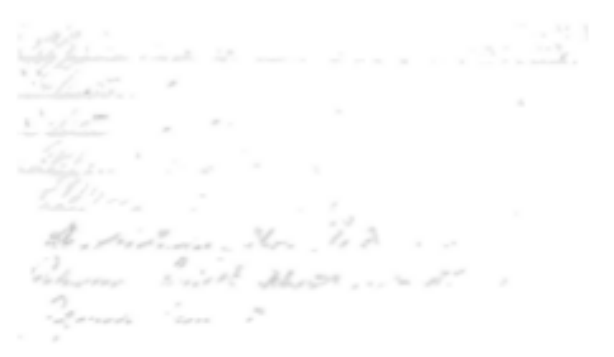

(a)

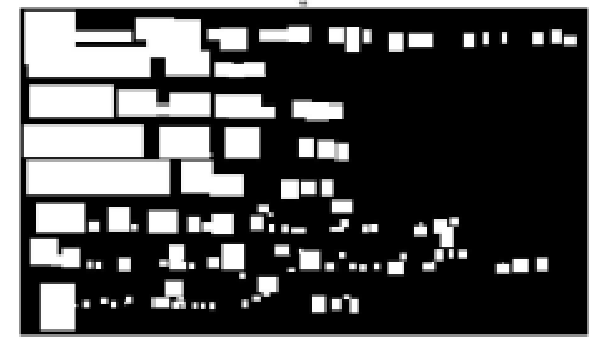

(c)

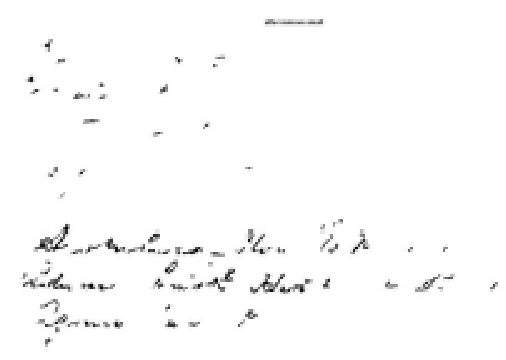

(b)

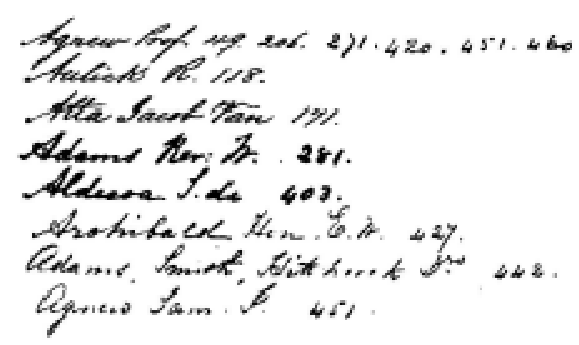

(d)

Figure 5. Faint text recovering for a high contrast image: (a) $R l$. (b) $R l$ binarization. (c) Rectangles fit object in $B I$. (d) $F I$.

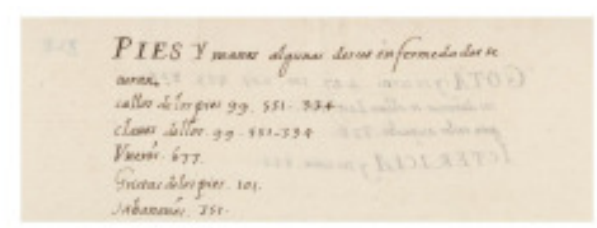

(a) $\mathrm{H} 08-14$



(c)

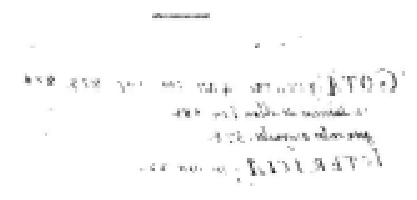

(b)

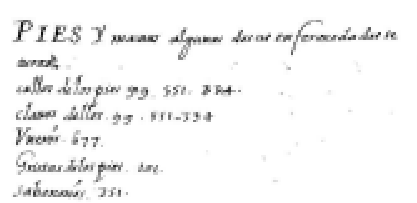

(d)

Figure 6. Shadow is avoided for a high contrast image: (a) $I$. (b) $R I$ binarization. (c) Rectangles fit object in $B I$. (d) $F I$.

\subsection{SHADOW REDUCTION}

Shadow pixels appear as small bright objects in the intensity image given by Eq.(7), that resulted by applying the BI as a mask on the gray scale. So we remove objects in the Int whose size multiplied by its intensity is less than the SW multiplied by the brightest pixel in text intensity. Figure 12 shows shadow reduction. 


$$
\operatorname{Int}(x, y)= \begin{cases}I(x, y), & B I(x, y)=0 \\ 255, & \text { Otherwise }\end{cases}
$$

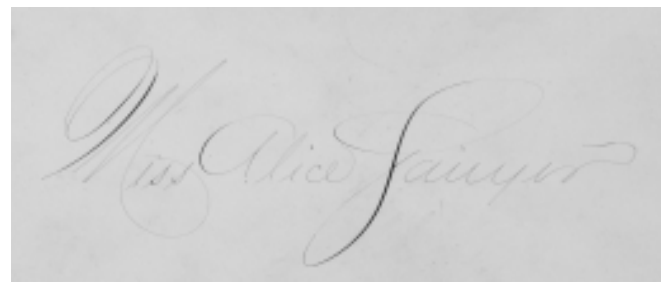

(a) HW7-13

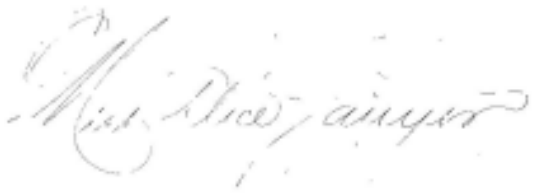

(c)

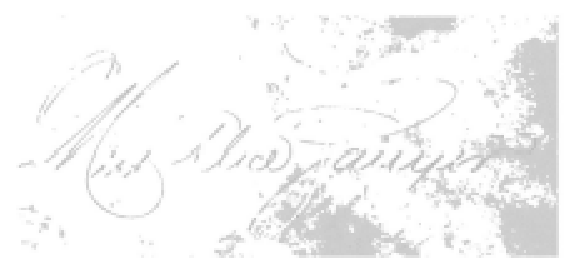

(b)

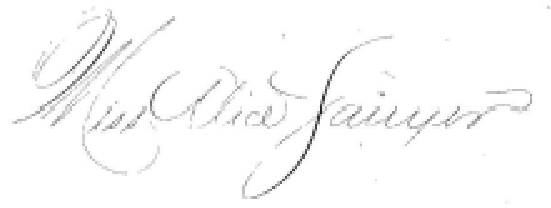

(d)

Figure 7. Faint text recovering for a high contrast image with very small $S W$ : (a)I. (b) $R 1$. (c) $R I$ binarization. (d) $F I$

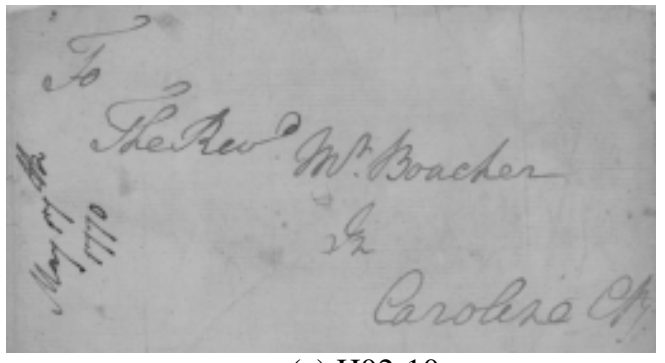

(a) $\mathrm{H} 02-10$

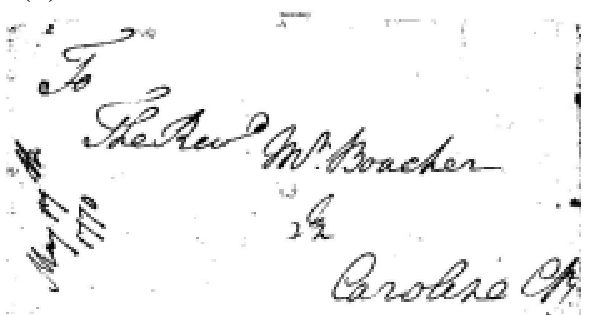

(b)

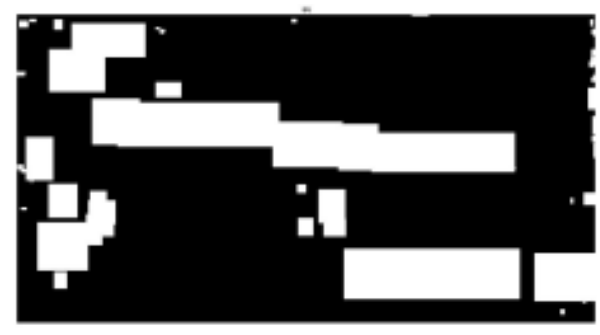

(c)

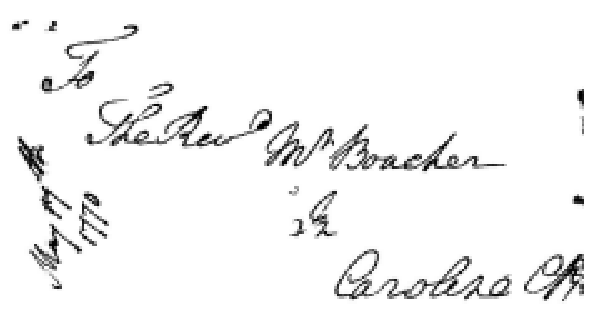

(d)

Figure 8. Noise removing for a low contrast image: (a) The gray scale image. (b) The binary image. (c) The rectangles fit each object in BI. (d) FI. 


\section{Experiments ANd Results}

The experiments are proceeded using DIBCO (9-10-11-12-13-14 and 16) series [17]. The proposed method is evaluated against recent methods using F-measure [18]. We propose three setups for the experiments with database. The first setup is for methods that display the average Fmeasure for all used datasets. The second setup is for methods that display the average F-measure for each used dataset separately. The third setup is for testing the proposed method using a dataset that my program has never seen before.

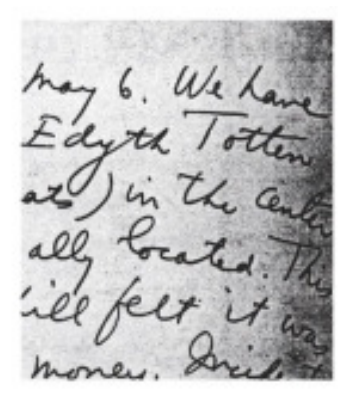

(a)

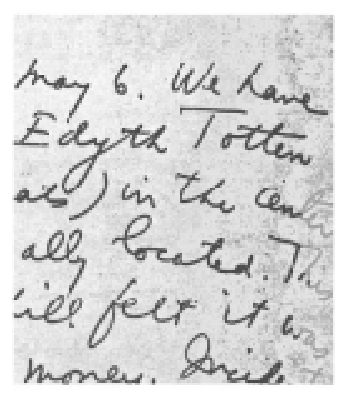

(b)

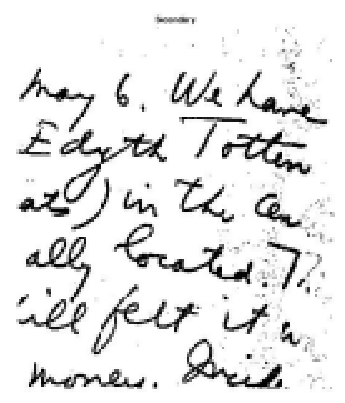

(c)

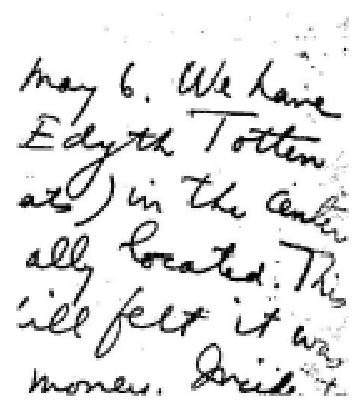

(f)

(d)

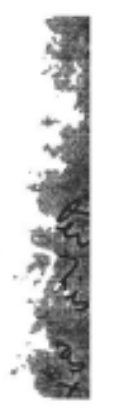

(e)

Figure 9. Recovering text lost during the normalization: (a) The gray scale image. (b) The normalized image. (c) The binary image. (d) R1. (e) R1 binarization. (f) The final image.

\subsection{Methods Display The Average F-Measure For All Used Database}

Kefali[12] and AL-Khatatneh[11] display the average F-measure for all used datasets. Kefali [12] used DIBCO (9-10-11and 12) datasets. The average F-measure of all these datasets is 85.7 and for the proposed method is 89.4. AL-Khatatneh [11] uses DIBCO (11 and 13). The average F-measure for printed and handwriting images are 87.6 and 79.6, respectively. In the proposed method they are 90.4 and 87.3 , respectively.

\subsection{Methods Display The Average F-Measure For Each Dataset USED SEPARATELY}

The proposed method is compared with Bataineh et al. [1] that uses DIBCO (9-10-11-12). Jetley [13], Nafchi [6], Lu [3] [6], Su [7] [6], Pratikakis [19][6], Moghaddam [4] [6] methods that use 
DIBC0(9-10-11), and Bataineh et al. [2] that uses DIBC0'09. The average F-measure for each database are presented in Table (1). Although many methods in literature have been proposed for degraded documents binarization, the main problem is that their performance is different according the dataset. The experimental results and comparison with recent algorithms show the effectiveness of our proposed method.

\subsection{METHODS PARTICIPATED IN ICFHR 2016 CONTEST}

My method recorded 86.7 for H-DIBCO 2016 [20], and the winner and the 12th method recorded 87.6 and 76.1 , respectively.

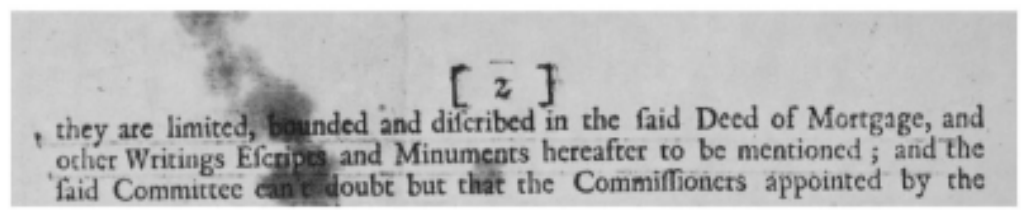

(a) P04-09

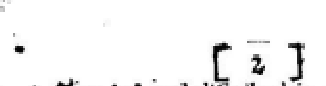

, they are limired, handed and difrribed in the faid Deed of Mortgage, and

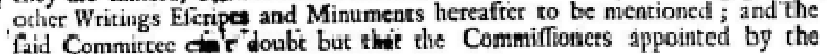

(b)

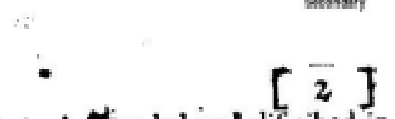

they are limited, thended and diferibed in the faid Deed of Mortgage, and

orher Writings Efcripe and Minuments hereafrer to be nentioned ; and the

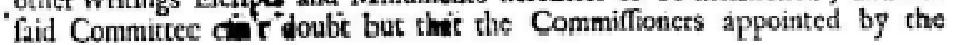

(c)

(d) 


\section{[2] \\ they are linited, bounded and diferibed in the aid Deed of Mortgage, and other Writiugs Eicripo and Minuments bereferer to be nentioned $;$ and the faid Commitee ar'r doubi but the the Commifioners appointed by the}

(e)

Figure 10. Stain objects detection: (a) I. (b) BI. (c) VMSSW . (d) The selected objects. (e) FI.

Figures $13,14,15,16,17$, and 18 show images suffer from compound challenges and their $F I$, that are used for visual analysis. Figure 13a suffers from stain. Its FI shows that the stain is removed while the text in this dark region is retained. Figure 14a contains shadow and stain. Its FI shows that shadow and stain are removed and text in the stain is retained. Image in Figure

15a suffers from shadow. FI for this image got rid of the shadow. Figure 16a shows an image suffers from faint text, non-uniform background, and stain. Its $F I$ shows that the non-uniform background and almost of stain are eliminated, and faint text is recovered. Figure 17a contains shadow and faint text. The $F I$ shows that the faint text is retrieved without shadow. Figure 18a shows an image contains non-uniform background. The background noise is eliminated in FI. Figure 19 show FI for images in Figures 4a, 4c, 4e, and 4g, respectively. Figure 19a shows that the faint text is retrieved. Figure 19b shows that the faint text is retrieved without shadow. Figure $19 \mathrm{c}$ shows that the shadow is removed. Figure 19d shows that almost of the faint text is retrieved without the background noise.

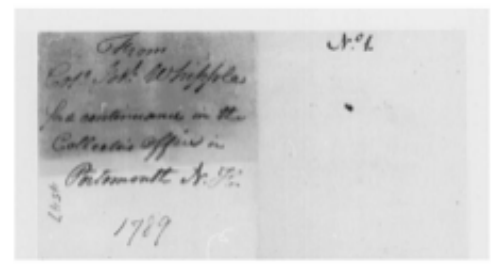

(a) $\mathrm{H} 05-09$

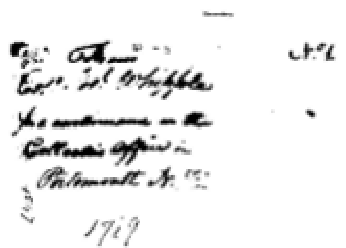

(b)

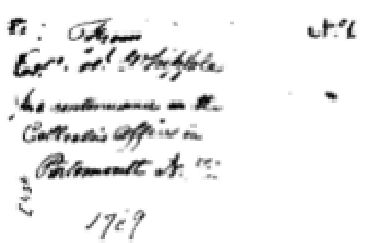

(c)

(d)

Figure 11. Bleeding through detection: (a) I. (b) BI. (c) Locations of objects surrounded by interference. (d) 


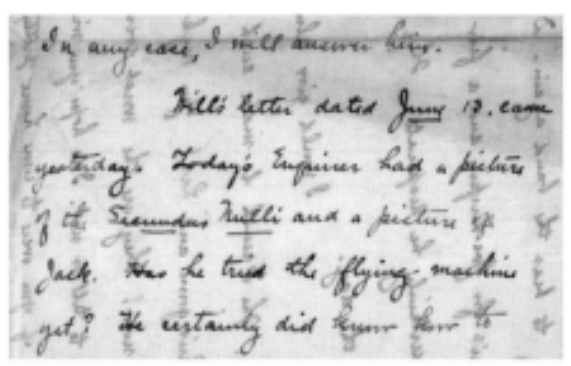

(a) H06-12

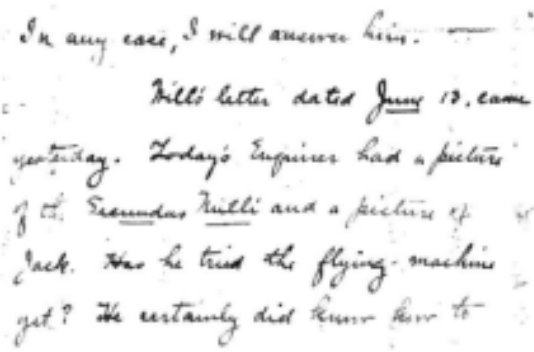

(c)

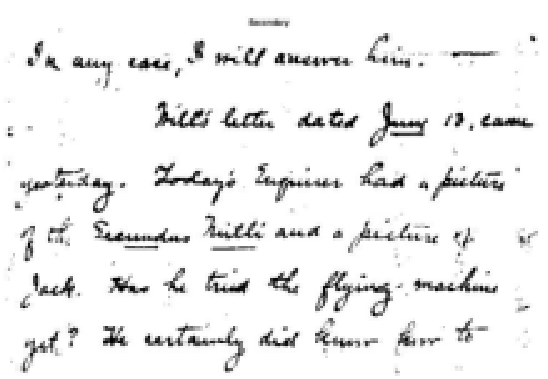

(b)

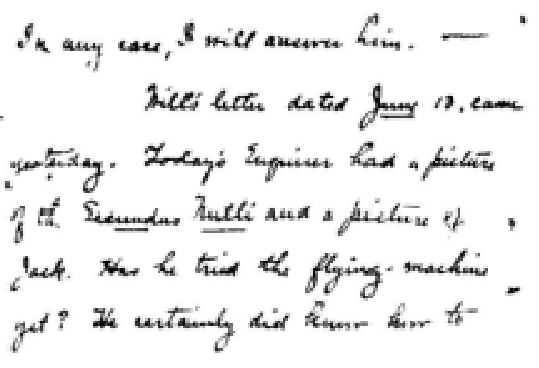

(d)

Figure 12. Shadow reduction: (a) I. (b) BI. (c) Int. (d) FI

Table 1. Average F-measure for DIBCO(09-10-11-12-13-14)

\begin{tabular}{|l|c|c|c|c|c|c|}
\hline Method & 09 & 10 & 11 & 12 & 13 & 14 \\
\hline Proposed & 90.7 & 89.1 & 88.9 & 88.7 & 88.9 & 93.3 \\
Bataineh et al.[1] & 89.3 & 84.7 & 87.2 & 88.1 & - & - \\
Jetley[13] & 77.0 & 84.6 & 83.5 & - & - & - \\
Nafchi[6] & 88.4 & 88.5 & 87.7 & - & - & - \\
Moghaddam[4] & 91.5 & 85.3 & 85.2 & - & - & - \\
Lu[3] & 91 & 86.4 & 81.6 & - & - & - \\
Su[7] & 91 & 85.4 & 85.5 & - & - & - \\
Pratikakis[19] & 90.3 & 86.7 & 83.58 & - & - & - \\
Shi[5] & 85.45 & - & - & - & - & - \\
Bataineh et al.[2] & 88 & - & - & - & - & - \\
\hline
\end{tabular}


Ravno za to nam je pa toliko treba političnega lista. Samo, da bi se moż zato pripraven nas̉el, kteri bi hotel teżavno vredaiśtvo na svoje rame vzeti. Dopisnikov, pravih iskrenih domoljubov, hi poznajo natanko stan našega ljudstva, mu se gotovo manjikalo ne bode. Oni spoznajo ime-

(a) PR5-13

Ravno za to nam je pa toliko treba političnega lista. Samo, da bi se moż are pripraven nas̉el, kteri bi hotel teżavno vredniétvo na evoje rame vzeti. Dopisnikov, pravih iskrenih domoljubov, ki poznajo natanko stan nas̉ega ljadstva, mu se gotovo manjkalo ne bode. Oni spoznajo ime-

(b)

Figure 13. An image suffers from stain and its $F I$

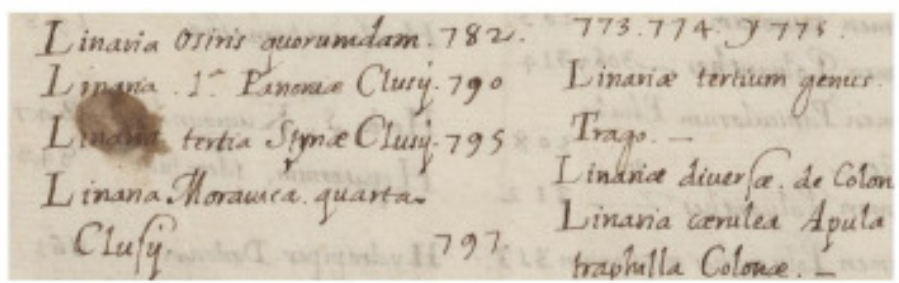

(a) $\mathrm{H} 07-14$

Linavia Orin's quoramdam.782. 773.774 .9775

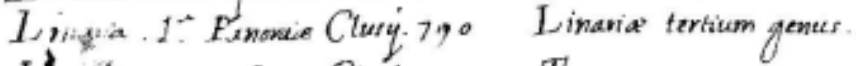

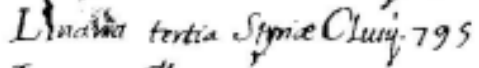

Linana. Horamis quartar Clufy.

797.

Trapo...

I inkina diuer C. de Coton Linaria cerrilea Apula trashilla Colonce. -

(b)

Figure 14. An image suffers from shadow and stain and its FI 


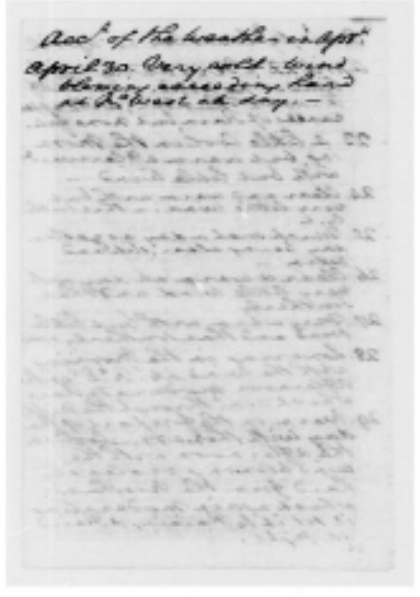

(a) H02-9

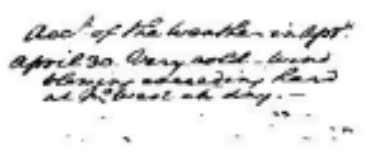

(b)

Figure 15. An image suffers from shadow and its FI

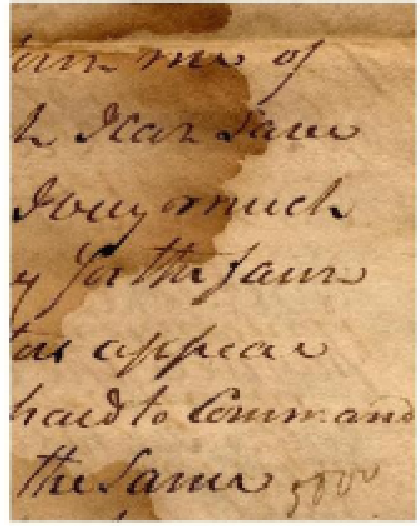

(a) HW4-11

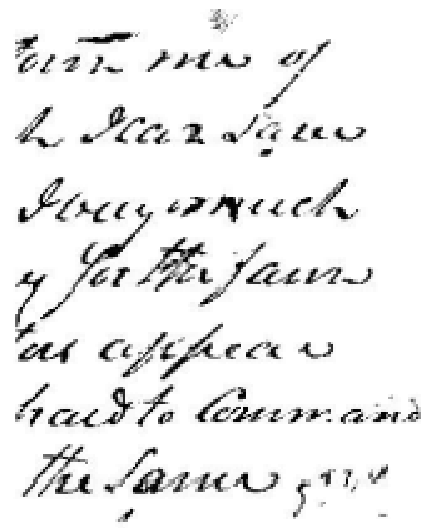

(b)

Figure 16. An image suffers from faint text, non-uniform background and stain, and its FI

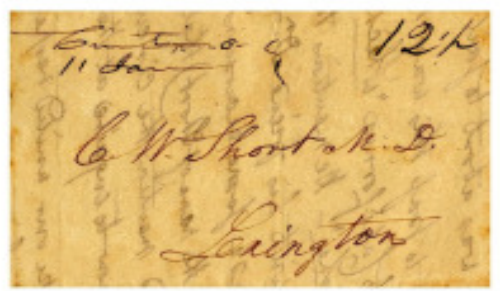

(a) HW7-11

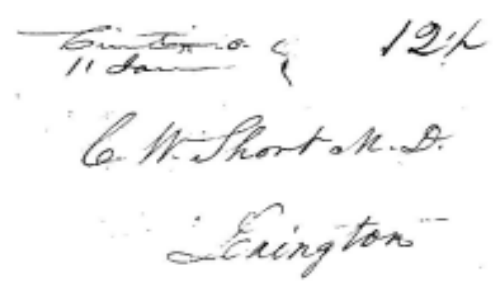

(b)

Figure 17. An image contains shadow and faint text, and its FI 


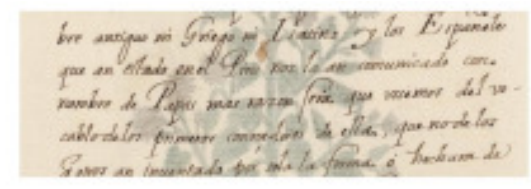

(a) $\mathrm{H} 09-14$

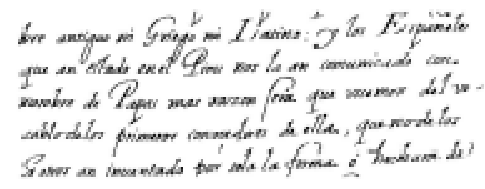

(b)

Figure 18. An image contains non-uniform background and its FI

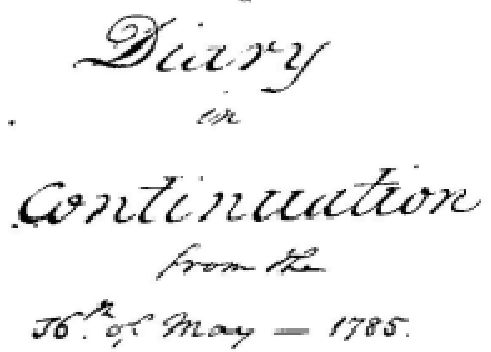

(a)

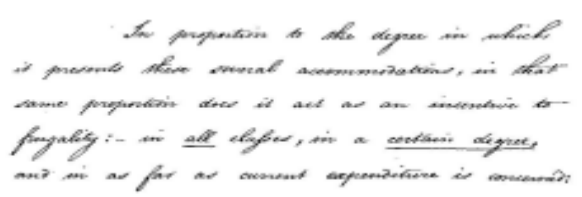

(c)

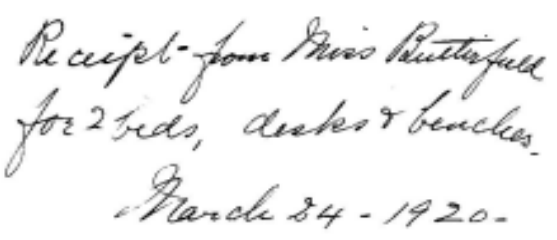

(b)

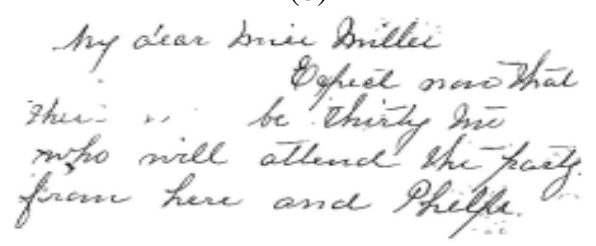

(d)

Figure 19. FI for images in figures $4 \mathrm{a}, 4 \mathrm{c}, 4 \mathrm{e}$, and $4 \mathrm{~g}$, respectively.

\section{CONCLUSION}

In this paper, a method for solving the binarization challenges is presented. The background of the investigated image is estimated and divided into three regions using Otsu multilevel technique. The difference between the average intensities of darkest and brightest regions of the estimated background is low in uniform background images and high in non-uniform background images. So that, the non-uniform backgrounds and almost of stains are detected and eliminated by normalization. The darkest background region is applied as a mask on the image for identifying the area that contains missing text. This area is binarized separately using a pseudo-local version of Otsu multilevel method for lost text recovering. Faint text, shadow or background noise for uniform background images are distinguished based on the image contrast. Thin or weak strokes are recovered. Stain and bleed-through objects are detected depending on estimated stroke width, and they are locally binarized using Otsu multilevel. Finally, a post processing step based on the estimated stroke width is applied to remove the remaining shadow. The main advantage of the proposed method is its robustness across all datasets. Our method couldn't remove shadow if it has the same contrast and stroke width of the text. For the non-uniform background images, it may find a difficulty to recover the extremely low contrast or very faint text exist in the darkest background region. 


\section{REFERENCES}

[1] B. Bataineh, S. N. H. S. Abdullah, and K. Omar, "Adaptive binarization method for degraded document images based on surface contrast variation," Pattern Analysis and Applications, vol. 20, no. 3, pp. 639-652, 2017.

[2] B. Bataineh, S. N. H. S. Abdullah, and K. Omar, "An adaptive local binarization method for document images based on a novel thresholding method and dynamic windows," Pattern Recognition Letters, vol. 32, no. 14, pp. 1805-1813, 2011.

[3]S. Lu, B. Su, and C. L. Tan, "Document image binarization using background estimation and stroke edges," International journal on document analysis and recognition, vol. 13, no. 4, pp. 303-314, 2010.

[4] R. F. Moghaddam and M. Cheriet, "Adotsu: An adaptive and parameterless generalization of Otsu's method for document image binarization," Pattern Recognition, vol. 45, no. 6, pp. 2419-2431, 2012.

[5] J. Shi, N. Ray, and H. Zhang, "Shape based local thresholding for binarization of document images," Pattern Recognition Letters, vol. 33, no. 1, pp. 24-32, 2012.

[6] H. Z. Nafchi, R. F. Moghaddam, and M. Cheriet, "Historical document binarization based on phase information of images," in Asian Conjerence on Computer Vision, pp. 1-12, Springer, 2012.

[7] B. Su, S. Lu, and C. L. Tan, "Binarization of historical document images using the local max-imum and minimum," in Proceedings oj the 9th IAPR International Workshop on Document Analysis Systems, pp. 159-166, ACM, 2010.

[8] N. R. Howe, "A Laplacian energy for document binarization," in Document Analysis and Recognition (ICDAR), 2011 International Conjerence on, pp. 6-10, IEEE, 2011.

[9] F. Jia, C. Shi, K. He, C. Wang, and B. Xiao, "Degraded document image binarization using structural symmetry of strokes," Pattern Recognition, vol. 74, pp. 225-240, 2018.

[10] Q. N. Vo, S. H. Kim, H. J. Yang, and G. Lee, "Binarization of degraded document images based on hierarchical deep supervised network," Pattern Recognition, vol. 74, pp. 568-586, 2018.

[11] A. M. AL-Khatatneh, S. A. Pitchay, and M. K. Al-qudah, "Compound binarization for degraded document images," ARPN Journal oj Engineering and Applied Sciences. ISSN, vol. 6608, pp. 594$599,2015$.

[12] A. Kefali, T. Sari, and H. Bahi, "Text/background separation in the degraded document im- ages by combining several thresholding techniques," WSEAS transactions on signal process- ing, vol. 10, pp. 436-443, 2014.

[13] S. Jetley, S. Belhe, V. K. Koppula, and A. Negi, "Two-stage hybrid binarization around fringe map based text line segmentation for document images," in Pattern Recognition (ICPR),2012 21st International Conjerence on, pp. 343-346, IEEE, 2012.

[14] J. Kittler and J. Illingworth, "On threshold selection using clustering criteria," IEEE transac- tions on systems, man, and cybernetics, no. 5, pp. 652-655, 1985. 
[15] Y.-T. Pai, Y.-F. Chang, and S.-J. Ruan, "Adaptive thresholding algorithm: Efficient computa- tion technique based on intelligent block detection for degraded document images," Pattern Recognition, vol. 43, no. 9, pp. 3177-3187, 2010.

[16] N. Otsu, "A threshold selection method from gray-level histograms," IEEE transactions on systems, man, and cybernetics, vol. 9, no. 1, pp. 62-66, 1979.

[17] K. Ntirogiannis, B. Gatos, and I. Pratikakis, "Icfhr2014 competition on handwritten docu- ment image binarization (h-dibco 2014)," in Frontiers in Handwriting Recognition (ICFHR), 2014 14th International Conjerence on, pp. 809-813, IEEE, 2014.

[18] O. Bojar, C. Buck, C. Federmann, B. Haddow, P. Koehn, J. Leveling, C. Monz, P. Pecina, M. Post, H. Saint-Amand, et al., "Findings of the 2014 workshop on statistical machine translation," in Proceedings oj the ninth workshop on statistical machine translation, pp. 12$58,2014$.

[19] I. Pratikakis, B. Gatos, and K. Ntirogiannis, "Icdar 2011 document image binarization con- test (DIBCO 2011)," in Document Analysis and Recognition (ICDAR), 2011 International Conjerence on, pp. 1506-1510, IEEE, 2011.

[20] I. Pratikakis, K. Zagoris, G. Barlas, and B. Gatos, "ICFHR2016 handwritten document image binarization contest (h-dibco 2016)," in 2016 15th International Conjerence on Frontiers in Handwriting Recognition (ICFHR), pp. 619-623, IEEE, 2016. 